\title{
The C-cavity, a highly versatile and simple laser design
}

\author{
Robert Lindberg' ${ }^{1}$, Fredrik Laurell ${ }^{1}$, Krister Fröjdh ${ }^{2}$ and Walter Margulis ${ }^{3}$ \\ 1. Department of Applied Physics, Royal Institute of Technology, 10691 Stockholm, Sweden \\ 2. Proximion AB, Skalholtsgatan 10, SE 16440 Kista, Sweden \\ 3. Department of Fiber Optics, RISE Acreo, 16440 Kista, Sweden \\ Authore-mail address: rl@laserphysics.kth.se
}

Electrically wavelength-tunable lasers employing chirped fiber Bragg gratings (CFBG) date back to 1993 [1], when a laser diode was connected to a CFBG and operated with a modulated driving current. Varying the modulation frequency thus enabled feedback from different regions of the CFBG, and hence wavelength-tuned operation. This linear cavity design was complemented by a ring variation, oftentimes referred to as a sigmacavity, five years later [2] in an Er-fiber laser. The sigma cavity configuration has since also been used in lasers employing semiconductor optical amplifiers (SOA) as a gain medium as well as a modulator [3].

As the wavelength tuning in both of these designs is accompanied by varying repetition rates, they are illsuited for applications relying on synchronization with other sources. It was not until 2017 that a wavelengthtunable design with a constant repetition rate was demonstrated [4]. This design used a fiber Bragg grating array (FBGA) instead of a CFBG to realize stepped tuning, and revolved around reflecting the circulating light from both ends of the FBGA. As the design was unidirectional, this was realized by connecting the FBGA to the midports of two circulators, thus equalizing the overall path through the FBGA, that fromed a ring cavity.

We present a simplified version of this design by employing a linear cavity configuration, which eliminates the need for two circulators [5]. Our laser is realized with a SOA, working as a gain medium and a modulator, and is schematically depicted in Fig. 1 a). The electrical gating sequence, consisting of a repeating pulse pair, that drives the SOA is shown in Fig. 1 b). The separations between these pulses correspond to the time it takes the light to exit the SOA, get reflected from the CFBG and return to the SOA in each arm -as indicated by the color coding. This gating sequence thus inhibits circular propagation, and we refer to the configuration as a $\mathrm{C}$ cavity -due to the resemblance to a "bent" linear cavity. Tuning is thus realized by simply varying the pulse separation by different fractions of the overall round-trip time. The tuning characteristic of this laser is presented in Fig. $1 \mathrm{c}$ ), from which it is seen that the tuning range extended $35 \mathrm{~nm}$-limited by the CFBG's reflection bandwidth. The linewidths were about $2 \mathrm{~nm}$, which could be reduced by using shorter gate pulses and/or a more strongly chirped grating. It should also be mentioned that configurations employing an FBGA instead of a CFBG results in linewidths primarily limited by the gratings' reflection bandwidths.

Despite the simple cavity configuration, this design enables highly versatile and tailorable means of operation by interleaving gating sequences. This not only enables average power scaling, but also time-multiplexed multiwavelength operation as illustrated in Fig. $1 \mathrm{~d}$ ). The gating sequences can also employ a common gate in one arm, which results in temporally split and overlapping optical pulses at the two outputs -as shown in Fig. 1 e). This immense operation flexibility makes the C-cavity design adaptable to a multitude of applications.

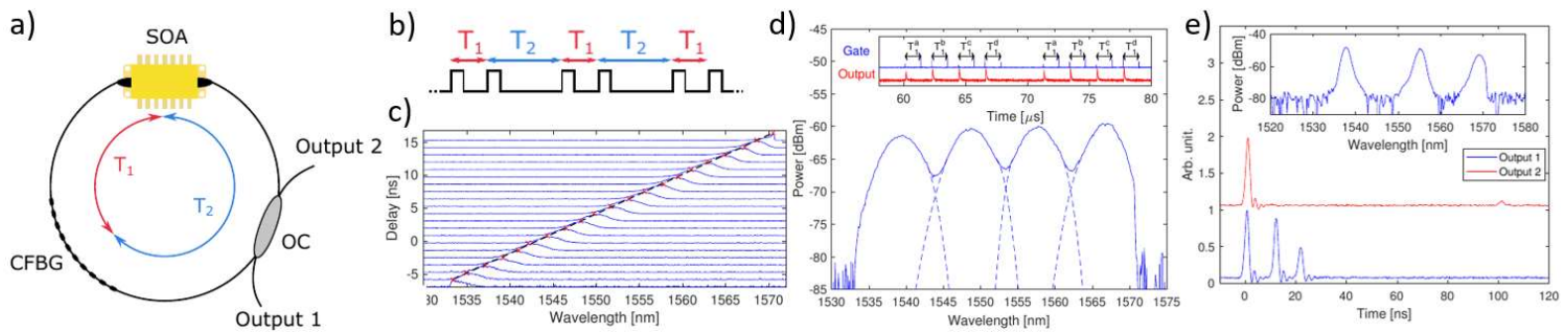

Fig. 1 A schematic depiction of the C-cavity layout is given in a) and the gating sequence shown in b). The achieved tuning characteristics are shown in c). Time-multiplexed four wavelength operation is demonstrated in d) and e) shows the output pulse sequences from the two outputs when using a common gate in one arm.

\section{References}

[1] P. A. Morton et al, "Mode-Locked Hybrid Soliton Pulse Source with Extremely Wide Operating Frequency Range," IEEE Photonics Technol. Lett. 5(1), 28-31 (1993).

[2] S. Li and K. T. Chan, "Electrical Wavelength-Tunable Actively Mode-Locked Fiber Ring Laser with a Linearly Chirped Fiber Bragg Grating," IEEE Photonics Technol. Lett. 10(6), 799-801 (1998).

[3] X. Yang el al, "Continuously tunable, narrow-linewidth laser based on a semiconductor optical amplifier and a linearly chirped fiber Bragg grating," Opt. Express 27, 14213-14220 (2019)

[4] T. Tiess, M. Becker, M. Rothhardt, H. Bartelt, and M. Jäger, "Discrete tuning concept for fiber-integrated lasers based on tailored FBG arrays and a theta cavity layout," Opt. Lett. 42(6), 1125-1128 (2017).

[5] R. Lindberg et al,, "C-cavity fiber laser employing a chirped fiber Bragg grating for electrically gated wavelength tuning," Opt. Express $28,9208-9215(2020)$ 